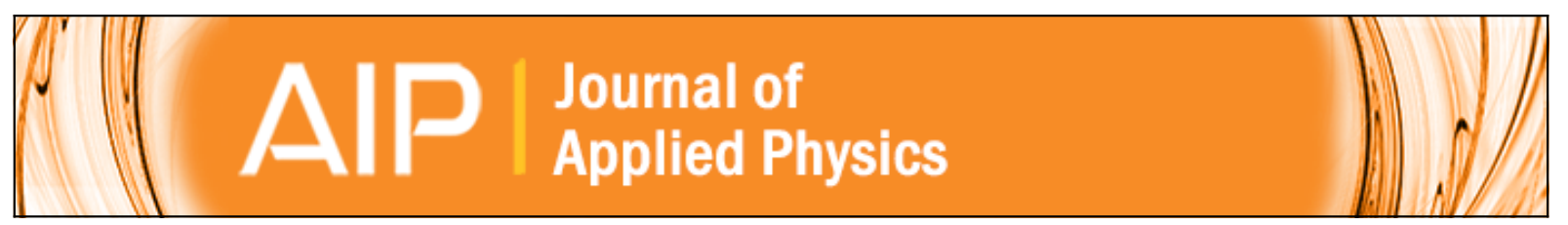

\title{
Microfabrication of magnetostrictive beams based on NiFe film doped with B and Mo for integrated sensor systems
}

A. Alfadhel, Y. Gianchandani, and J. Kosel

Citation: Journal of Applied Physics 111, 07 E515 (2012); doi: 10.1063/1.3679016

View online: http://dx.doi.org/10.1063/1.3679016

View Table of Contents: http://scitation.aip.org/content/aip/journal/jap/111/7?ver=pdfcov

Published by the AIP Publishing

\section{Articles you may be interested in}

Meander-shaped magnetoimpedance sensor for measuring inhomogeneous magnetic fringe fields of NiFe films Appl. Phys. Lett. 105, 172404 (2014); 10.1063/1.4900786

Development of FeNiMoB thin film materials for microfabricated magnetoelastic sensors

J. Appl. Phys. 112, 113912 (2012); 10.1063/1.4768458

Actuation micro-design based on martensitic phase transformations in shape memory alloys

AIP Conf. Proc. 1472, 135 (2012); 10.1063/1.4748080

Patterned L10-FePt for polarization of magnetic films

J. Appl. Phys. 109, 07 A720 (2011); 10.1063/1.3561172

Effect of heat treatment on microstructure and magnetic properties of anisotropic $\mathrm{Nd}-\mathrm{Fe}-\mathrm{B}$ films with $\mathrm{Mo}$ or $\mathrm{Ti}$ buffer layer

J. Appl. Phys. 98, 113905 (2005); 10.1063/1.2136208

MIT LINCOLN

LABORATORY CAREERS

Discover the satisfaction of innovation and service

to the nation
- Space Control

- Air \& Missile Defense

- Communications Systems \& Cyber Security

- Intelligence, Surveillance and

Reconnaissance Systems

- Advanced
Electronics
- Tactical Systems
" Homeland
Protection
- Air Traffic Control

LINCOLN LABORATORY

MassachusetTS Institute OF TeChNOLOGY

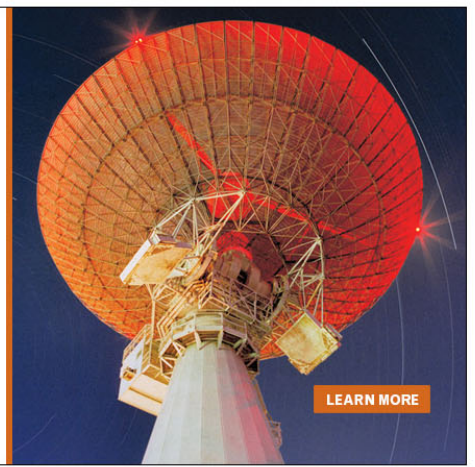




\title{
Microfabrication of magnetostrictive beams based on NiFe film doped with $B$ and Mo for integrated sensor systems
}

\author{
A. Alfadhel, ${ }^{1, a)}$ Y. Gianchandani, ${ }^{2}$ and J. Kosel ${ }^{1}$ \\ ${ }^{1}$ Division of Physical Sciences and Engineering, King Abdullah University of Science and Technology, \\ Thuwal 23955, Saudi Arabia \\ ${ }^{2}$ Department of Electrical Engineering and Computer Science, University of Michigan, Ann Arbor, \\ Michigan 48109, USA
}

(Presented 1 November 2011; received 11 October 2011; accepted 30 November 2011; published online 9 March 2012)

This paper reports the development of integrated micro-sensors consisting of 1 - $\mu \mathrm{m}$-thick magnetostrictive cantilevers or bridges with $500 \mu \mathrm{m}$ in length and conducting interrogation elements. The thin films are fabricated by sputter deposition of NiFe doped with B and Mo, and the magnetic properties are enhanced by field annealing, resulting in a coercivity of $2.4 \mathrm{Oe}$. In operation, an alternating current applied to the interrogation elements magnetizes the magnetostrictive structures. The longitudinal resonant frequency is detected as an impedance change of the interrogation elements. The magnetostrictive micro-beams provide high resonant frequencies-2.95 MHz for the cantilever and $5.46 \mathrm{MHz}$ for the bridge - which can be exploited to develop sensors of high sensitivity. (C) 2012 American Institute of Physics. [doi:10.1063/1.3679016]

\section{INTRODUCTION}

High-performance resonating sensors have attracted interest for many MEMS applications, especially in the field of biological species detection. ${ }^{1}$ For such applications, the surface of the sensor is functionalized to specifically bind to a target analyte. Binding of the target causes a change in the resonant frequency, which, in turn, enables analyte detection and quantification. ${ }^{2}$ Recently, devices that utilize magnetostrictive materials for sensing have been investigated. Compared to other options, such as silicon cantilevers, these vibrate in longitudinal direction instead of transverse direction, leading to higher resonant frequencies. This offers the potential advantage of higher sensitivity.

Previous researches showed that magnetostrictive sensors in the form of beams have a high signal quality factor in air, with reasonable signal damping in higher viscosity fluids. ${ }^{2,8}$ Such sensors have been successfully used for physical, chemical, and biological sensing. ${ }^{3-6}$ The materials typically used for magnetostrictive sensor applications are amorphous magnetostrictive ribbons of about $25 \mu \mathrm{m}$ thickness fabricated by melt spinning. So far, these sensors have been operated by external coils for actuation and sensing. This approach does not easily allow integration using a standard microfabrication process, and their potential for miniaturization is limited. Further miniaturization, though, can be expected to yield an increase in sensitivity, since the resonant frequency scales with the inverse of the sensor size. The main challenges for the development of magnetostrictive sensor systems in the micro-scale regime are the fabrication of soft magnetic thin films with high magnetostriction and obtaining a high signal-to-noise ratio. The latter can only be achieved if the interrogation elements are in close proximity with the magnetostrictive material. In this paper, we report the

${ }^{a)}$ Electronic mail: ahmed.fadhel@kaust.edu.sa. fabrication of magnetostrictive fixed-free (cantilever) and fixed-fixed (bridge) beams and their integration with microstructures for interrogation on a common chip. The fabricated magnetostrictive material is similar to the commercially available Metglas $2826 \mathrm{MB}$ that is characterized by a very small coercivity, which makes it easy to magnetize, and large magnetomechanical coupling, which provides a large mechanical response upon application of a magnetic field. The developed sensor system enables, for the first time, the detection of the resonant frequency of magnetostrictive micro-beams on an integrated device.

\section{METHODS}

\section{A. Sensor design}

The sensor system consists of a magnetostrictive cantilever or bridge of length $500 \mu \mathrm{m}$, width $100 \mu \mathrm{m}$, and thickness $1 \mu \mathrm{m}$ fabricated $2 \mu \mathrm{m}$ above the interrogation elements, which are 23 conducting lines of $10 \times 0.5 \mu \mathrm{m}^{2}$ arranged parallel to each other with a pitch of $10 \mu \mathrm{m}$ (Fig. 1). The magnetostrictive beams are actuated by the magnetic field produced by the current $I_{\mathrm{AC}}$ applied to the interrogation elements. With $I_{A C} 20$ $\mathrm{mA}$, a magnetic field of 0.5 Oe per conducting line is produced $2 \mu \mathrm{m}$ above the interrogation elements. The beam response depends, besides others, on the actuation frequency and will be highest at the resonant frequency.

The longitudinal resonant frequency for the fixed-fixed beams (Eq. (1) $)^{7}$ and fixed-free beams (Eq. (2) $)^{7}$ can be evaluated as follows:

$$
f=\frac{1}{2 L} \sqrt{\frac{\mathrm{E}}{\rho(1-\nu)}},
$$

and

$$
f=\frac{1}{4 L} \sqrt{\frac{\mathrm{E}}{\rho(1-\nu)}},
$$




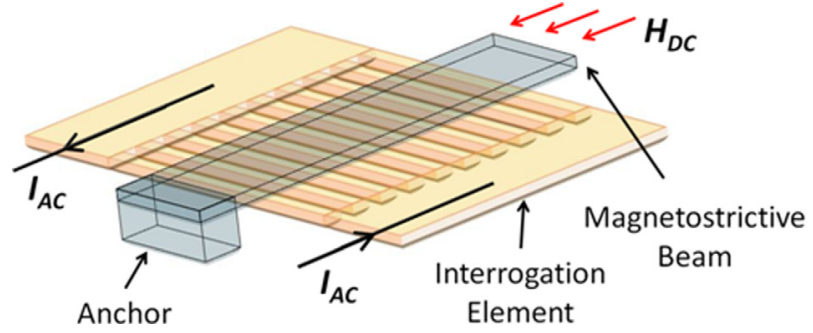

FIG. 1. (Color online) Illustration of the sensor system consisting of a cantilever beam above interrogation elements. An AC current provides actuation of the cantilever and a DC magnetic field is applied in the longitudinal direction of the beam to set the working point.

where $L$ is the length, $E$ is Young's modulus, $\rho$ is the density, and $\nu$ is Poisson's ratio. The Young's modulus for the magnetostrictive material is $100-110 \mathrm{GPa}$, and the density is $7.54 \mathrm{~g} / \mathrm{cm}^{3}$. Loading the beam with a mass, for example, a biological target analyte, will change the effective value of $\rho$. Due to the inverse magnetostrictive effect, the vibration of the beam causes a change of the permeability, which is sensed by the interrogation elements as a change in impedance. Therefore, the impedance of the interrogation elements is used to determine the resonant frequency of the beams, which contains the sensing information.

Applying a DC bias field $H_{\mathrm{DC}}$ in the longitudinal direction enhances the response of the magnetostrictive beams, which is typically highest around the knee of the magnetization curve. When applying $H_{\mathrm{DC}}$ to a magnetostrictive material, the Young's modulus changes ${ }^{8}$ such that

$$
E\left(H_{D C}\right)=E_{o}+\varphi\left(H_{D C}\right)
$$

where $H_{\mathrm{DC}}$ is the applied DC magnetic field, $E\left(H_{\mathrm{DC}}\right)$ is the varying Young's modulus, $E_{o}$ is the zero field Young's modulus, and $\varphi$ is a nonlinear function that relates $H_{\mathrm{DC}}$ to the stiffness change. This effect yields a dependence of the resonant frequency on $H_{\mathrm{DC}}$, and it is, therefore, important for $H_{\mathrm{DC}}$ to be homogeneous and constant throughout the measurement.

\section{B. Microfabrication process}

The developed fabrication process is completed on standard silicon wafers. Patterning of the structures is done through photolithography. First, the interrogating microstructure is patterned. Reactive ion etching is used to etch the Si wafer, and, then the trenches are filled with $500 \mathrm{~nm}$ of gold through sputter deposition. The reason for having the interrogating microstructures integrated in the substrate is to reduce the conformal growth for the following layers. A silicon nitride $\left(\mathrm{Si}_{3} \mathrm{~N}_{4}\right)$ film is deposited to provide electrical isolation between the interrogating elements and the beams. It also serves as an etch stop layer when releasing the beams. In the next step, $2 \mu \mathrm{m}$ of amorphous silicon are deposited with PECVD to be used as the sacrificial layer. In order to create the anchors, the amorphous $\mathrm{Si}$ is etched down to the $\mathrm{Si}_{3} \mathrm{~N}_{4}$ film through the use of reactive ion etching. The beams are patterned through bi-layer lift-off lithography to allow easy lift-off with clean edges. The magnetostrictive film fabrication

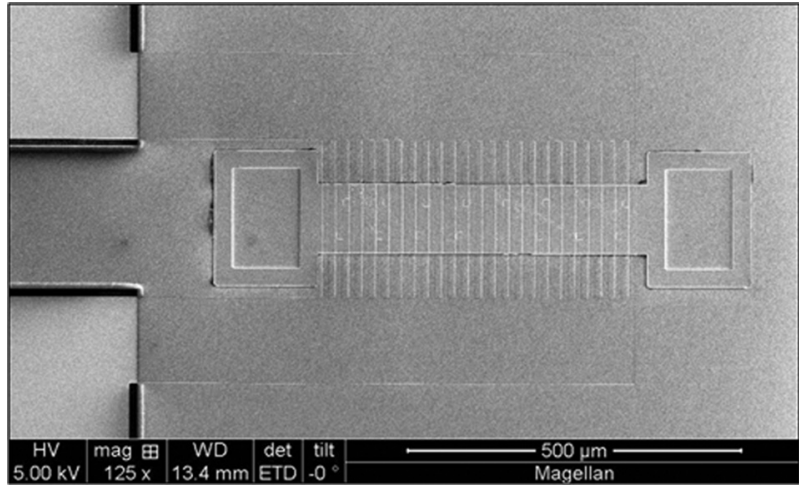

FIG. 2. SEM image of a $500-\mu \mathrm{m}$-long bridge with interrogation structure underneath.

has been optimized in several steps toward low coercivity and is deposited through co-sputtering of $\mathrm{Ni}_{50} \mathrm{Fe}_{50}$, boron (B), and molybdenum (Mo) targets. DC power is used for $\mathrm{Ni}_{50} \mathrm{Fe}_{50}$ $(200 \mathrm{~W})$ and Mo $(55 \mathrm{~W})$ targets, and RF power is used for B $(110 \mathrm{~W})$. To open the gold contact pads of the interrogation elements, a patterning and etching step through amorphous $\mathrm{Si}$ and the $\mathrm{Si}_{3} \mathrm{~N}_{4}$ film is performed using reactive ion etching. Finally, the beams are released by etching the amorphous $\mathrm{Si}$ sacrificial film using $\mathrm{XeF}_{2}$ vapor phase etching. Scanning electron microscopy (SEM), x-ray photoelectron spectroscopy (XPS), and vibrating sample magnetometry (VSM) are used to characterize the properties of the deposited material.

\section{EXPERIMENTS AND DISCUSSION}

\section{A. Fabrication results}

Fig. 2 shows a $500-\mu \mathrm{m}$-long bridge fabricated with the developed process. Using XPS, the composition of the deposited magnetic film is found to be $\mathrm{Fe}_{45} \mathrm{Ni}_{31} \mathrm{Mo}_{10} \mathrm{~B}_{13}$. Some topography of the interrogation elements can be observed at the beam surface. The conductive stack deposited into the $\mathrm{Si}$ trenches was $90 \mathrm{~nm}$ less than the trench depth and, e.g., mechanical polishing would be required before the $\mathrm{Si}_{3} \mathrm{~N}_{4}$ deposition.

\section{B. Magnetic properties}

Fig. 3 and Fig. 4 show the magnetization curves in longitudinal and transverse direction, respectively, of a

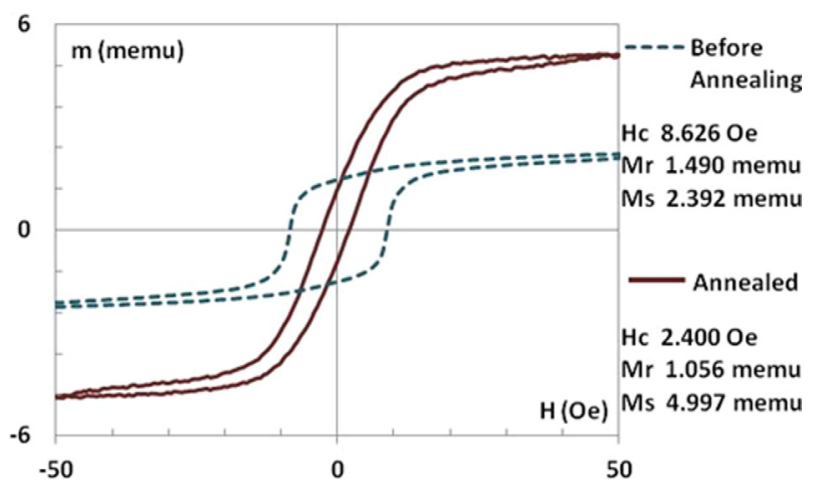

FIG. 3. (Color online) Magnetization curves of a 500 - $\mu \mathrm{m}$-long cantilever in longitudinal direction before and after annealing. 


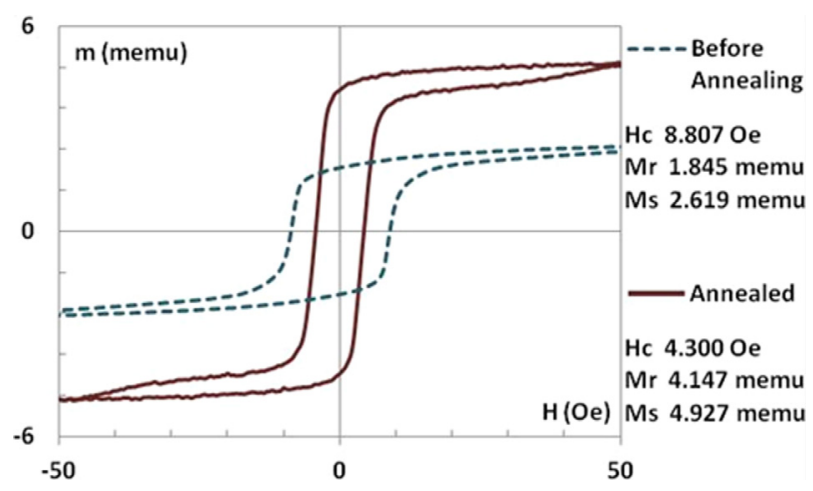

FIG. 4. (Color online) Magnetization curves of a $500-\mu \mathrm{m}$-long cantilever in transverse direction before and after annealing.

cantilever before and after field annealing. The as-prepared films are almost isotropic, with a coercivity around 8.6 Oe. After field annealing in the longitudinal direction at $350^{\circ} \mathrm{C}$ and $1000 \mathrm{Oe}$, an induced anisotropy was observed and the coercivity decreased to $2.4 \mathrm{Oe}$ in the longitudinal direction and $4.3 \mathrm{Oe}$ in the transverse direction. The remanence in longitudinal direction became lower after annealing, while the remanence in the transverse direction increased.

\section{Resonant frequency measurement}

The resonant frequency of the beams is detected by measuring the $\mathrm{AC}$ impedance of the interrogation elements. The impedance is measured with an impedance analyzer, applying an AC current $I_{\mathrm{AC}}$ of amplitude $20 \mathrm{~mA}$ to the interrogation element while sweeping the frequency. An external DC magnetic field $H_{\mathrm{DC}}$ of 20 Oe is applied in the longitudinal direction using Helmholtz coils to bias the magnetostrictive sensor to its point of highest sensitivity. The impedances measured for the cantilever and the bridge are shown in Fig. 5 and Fig. 6, respectively. At the resonant frequencies, the impedances exhibit an abrupt change of about $0.1 \Omega$. The resonant frequencies are found to be $2.95 \mathrm{MHz}$ for the cantilever and 5.46 MHz for the bridge. From Eq. (1) and Eq. (2), the theoretical values of the resonant frequencies are about 4.6 MHz in the case of the bridge and about $2.3 \mathrm{MHz}$ in the case of the cantilever. These values are smaller than the ones

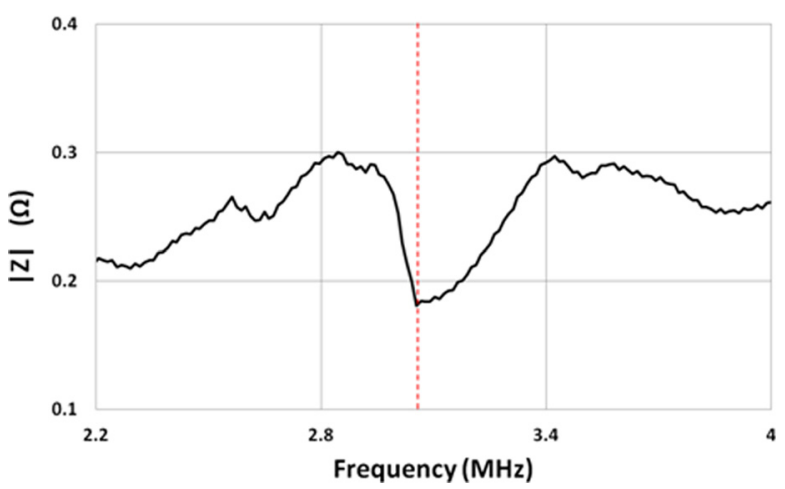

FIG. 5. (Color online) Impedance as a function of the frequency measured for a $500-\mu \mathrm{m}-$ long cantilever.

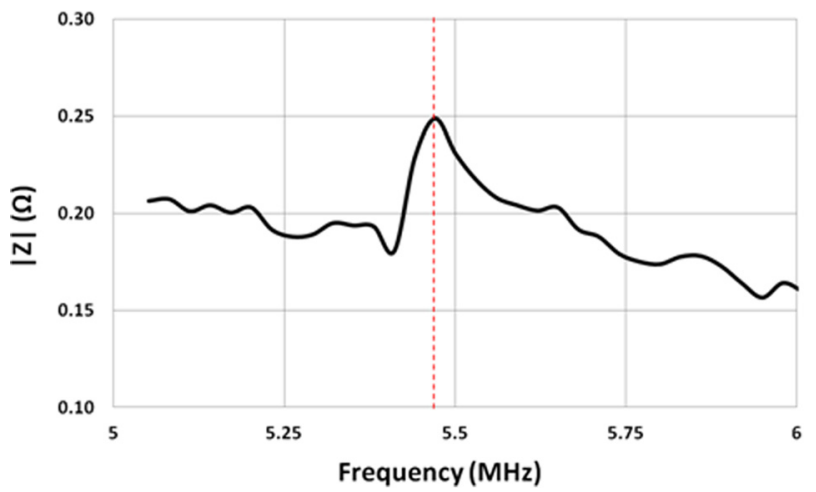

FIG. 6. (Color online) Impedance as a function of the frequency measured for a $500-\mu \mathrm{m}$-long bridge.

found experimentally. This is due to the effect of $H_{\mathrm{DC}}$ that increases the Young's modulus (see Eq. (3)), which, in turn, yields an increase of the resonant frequency. The resonant frequencies of the cantilever and the bridge are not exactly multiple of each other, which could be due to differences in the effective lengths of the beams. Further studies are conducted to investigate the effect of other vibration modes on the beam longitudinal resonant frequency.

\section{CONCLUSION}

A new sensor system using magnetostrictive microbeams and integrated interrogation elements was reported. A thin film process for fabricating soft magnetic, magnetostrictive sensor materials has been developed, including co-sputtering of $\mathrm{FeNi}, \mathrm{Mo}$, and $\mathrm{B}$ and field annealing to release stress and induce magnetic anisotropy. The integration of $500-\mu \mathrm{m}$-long magnetostrictive cantilevers and bridges with conducting interrogation elements is described. The impedance of the interrogation elements clearly showed the resonant frequencies of the magnetostrictive beams, which could be utilized for sensor applications.

\section{ACKNOWLEDGMENTS}

This work was supported by the KAUST Global Collaborative Research program. The authors acknowledge the support from the KAUST advanced nanofabrication facility (KANF), especially Ahad Sayed and Dr. Zhihong Wang.

${ }^{1}$ M. Ramasamy, C. Liang, and B. C. Prorok, MEMS and Nanotechnology: Proceedings of the 2010 Annual Conference on Experimental and Applied Mechanics, Indianapolis, Indiana 2010, edited by Tom Proulx (Springer, New York), Vol. 2, p. 9.

${ }^{2}$ J. Wan, H. Shu, S. Huang, B. Fiebor, I.-H. Chen, V. Petrenko, and B. A. Chin, IEEE Sens. J. 7, 470 (2007).

${ }^{3}$ C. A. Grimes, K. G. Ong, K. Loiselle, P. G. Stoyanov, and D. Kouzoudis, Smart Mater. Struct. 8, 639 (1999).

${ }^{4}$ C. Mungle, C. A. Grimes, and W. R. Dreschel, Sens. Actuators 101, 143 (2002).

${ }^{5}$ C. Ruan, K. Zeng, O. K. Varghese, and C. A. Grimes, Anal. Chem. 75, 6494 (2003).

${ }^{6}$ C. Ruan, K. Zeng, O. K. Varghese, and C. A. Grimes, Biosens. Bioelectron. 20, 585 (2004).

${ }^{7}$ C. Liang, Appl. Phys. Lett. 90, 221912 (2007).

${ }^{8}$ S. Green and Y. Gianchandani, J. Microelectromech. Syst. 18, 64 (2009). 